\title{
Use of GLP-1 mimetic in type 2 diabetes mellitus: is it the end of fragility fractures?
}

\author{
Guillaume Mabilleau
}

Received: 3 October 2014/ Accepted: 18 October 2014/Published online: 28 October 2014

(C) Springer Science+Business Media New York 2014

Type 2 diabetes mellitus (T2DM) is an insidious disease characterized by hyperglycemia and hyperinsulinemia. Among all complications of T2DM, bone fractures represent a severe alteration of the quality of life of affected individuals and are cost-effective. One explanation for bone fracture could be linked to other diabetes complications such as retinopathy and neuropathy, which increase the incidence of falls. Another explanation could reside in a direct effect of T2DM on bone resulting in poor "quality" of the bone matrix.

GLP-1 is a gut hormone released in the blood stream rapidly after a meal that potentializes insulin secretion from the pancreatic beta-cells [1]. However, in the blood stream, GLP-1 is rapidly inactivated by the dipeptidyl peptidase-4 (DPP4) enzyme [2]. As such two strategies have been applied by the pharmaceutical industries to target the GLP1 pathway: generation of injectable longer-acting GLP-1 receptor agonists (GLP-1Ra) and the development of DPP4 inhibitors (DPP4i). An unexpected role of GLP-1 in controlling bone strength in healthy animal model has recently been highlighted by our group suggesting that the GLP-1 pathway might also be important for an optimal bone matrix quality [3].

G. Mabilleau ( $\square)$

GEROM Groupe Etudes Remodelage Osseux et bioMatériaux UPRES EA 4658 LHEA, IRIS-IBS Institut de Biologie en Santé, CHU d'Angers, LUNAM Université, 4 rue Larrey,

49933 Angers Cedex 09, France

e-mail: guillaume.mabilleau@univ-angers.fr

G. Mabilleau

SCIAM, Service Commun d'Imagerie et Analyses

Microscopiques, IRIS-IBS Institut de Biologie en Santé, CHU

d'Angers, LUNAM Université, 49933 Angers Cedex, France
As such, the idea of using GLP-1 mimetic in order to reduce the occurrence of bone fracture in T2DM is tempting. However, conflicting results have been reported regarding the efficacy of GLP-1 mimetic in reducing fracture risk in T2DM. Monami et al. conducted a metaanalysis of randomized clinical trials [4] and reported a reduction in fracture risk in DPP4i users as compared with other antidiabetic drugs. However, few months ago, Driessen and coworkers [5] conducted a retrospective population-based cohort study, using data from the clinical practice research datalink database, one of the largest databases in Europe, and failed to show any beneficial effect of DPP4i in reducing fracture risk as compared with other antidiabetic drugs. Conflicting results have also been reported with the use of GLP-1Ra. Indeed, earlier this year, our group conducted a meta-analysis of randomized clinical trials in GLP-1Ra users [6]. We failed to demonstrate any beneficial role of GLP-1Ra use in reducing fracture risk. In the present issue of Endocrine, Su and coworkers report a reduction in fracture risk in GLP-1Ra users as compared with other antidiabetic drugs [7]. The discrepancy between our study and those of Su et al. resides in the number of included studies with fewer studies included in our meta-analysis based on study duration $>24$ weeks.

The observed conflicting results on the beneficial effects of GLP-1 mimetics in reducing bone fracture may reside in the fact that randomized clinical trials have not been designed for the assessment of bone fractures. Indeed, often bone fractures were disclosed as serious adverse events, although this probably represents only a fraction of all fractures. Indeed, only non-vertebral fractures were reported in the aforementioned studies. Vertebral fractures are often asymptomatic and largely undiagnosed and require a radiological and morphometrical approach to truly evaluate their incidence in population studies. Previous investigation 
using a radiological and morphometrical approach was conducted in secondary osteoporosis (growth hormone deficiency, acromegaly) or thaizolidinedione-treated diabetic patients and reported a significant prevalence of vertebral fractures [8-10]. In this context, the effect of GLP-1 mimetics on vertebral fracture risk is largely unknown. This highlights the critical need for a specific study/trial that would be adequately powered to assess skeletal health in T2DM patients, before assumption can be made regarding the beneficial effect of GLP-1 mimetics on skeletal health in T2DM.

\section{References}

1. L.L. Baggio, D.J. Drucker, Biology of incretins: GLP-1 and GIP. Gastroenterology 132, 2131-2157 (2007)

2. C.F. Deacon, A.H. Johnsen, J.J. Holst, Degradation of glucagonlike peptide- 1 by human plasma in vitro yields an $\mathrm{N}$-terminally truncated peptide that is a major endogenous metabolite in vivo. J. Clin. Endocrinol. Metab. 80, 952-957 (1995)

3. G. Mabilleau, A. Mieczkowska, N. Irwin, P.R. Flatt, D. Chappard, Optimal bone mechanical and material properties require a functional glucagon-like peptide-1 receptor. J. Endocrinol. 219, 59-68 (2013)

4. M. Monami, I. Dicembrini, A. Antenore, E. Mannucci, Dipeptidyl peptidase-4 inhibitors and bone fractures: a meta-analysis of randomized clinical trials. Diabetes Care 34, 2474-2476 (2011)

5. J.H. Driessen, H.A. van Onzenoort, R.M. Henry, A. Lalmohamed, J.P. van den Bergh, C. Neef et al., Use of dipeptidyl peptidase-4 inhibitors for type 2 diabetes mellitus and risk of fracture. Bone 68, 124-130 (2014)

6. G. Mabilleau, A. Mieczkowska, D. Chappard, Use of glucagonlike peptide- 1 receptor agonists and bone fractures: a meta-analysis of randomized clinical trials. J Diabetes 6, 260-266 (2014)

7. B. Su, H. Sheng, M. Zhang, L. Bu, P. Yang, L. Li et al., Risk of bone fractures associated with glucagon-like peptide-1 receptor agonists' treatment: a meta-analysis of randomized controlled trials. Endocrine (2014). doi:10.1007/s12020-014-0361-4

8. T. Mancini, G. Mazziotti, M. Doga, R. Carpinteri, N. Simetovic, P.P. Vescovi et al., Vertebral fractures in males with type 2 diabetes treated with rosiglitazone. Bone 45, 784-788 (2009)

9. G. Mazziotti, A. Bianchi, S. Bonadonna, V. Cimino, I. Patelli, A. Fusco et al., Prevalence of vertebral fractures in men with acromegaly. J. Clin. Endocrinol. Metab. 93, 4649-4655 (2008)

10. G. Mazziotti, A. Bianchi, S. Bonadonna, M. Nuzzo, V. Cimino, A. Fusco et al., Increased prevalence of radiological spinal deformities in adult patients with GH deficiency: influence of GH replacement therapy. J. Bone Miner. Res. 21, 520-528 (2006) 